\title{
Chaotic Attractors of Two-dimensional Invertible Maps
}

\author{
VADIM S. ANISHCHENKO *, TATJANA E. VADIVASOVA, GALINA I. STRELKOVA \\ and ANDREY S. KOPEIKIN \\ Nonlinear Dynamics Laboratory, Department of Physics, Saratov State University, Astrakhanskaya str. 83, 410026 Russia
}

(Received 20 March 1998)

\begin{abstract}
In this paper, we investigate the characteristics of quasihyperbolic attractors and quasiattractors in invertible dissipative maps of the plane. The criteria which allow one to diagnose the indicated types of attractors in numerical experiments are formulated.
\end{abstract}

Keywords: Attractor, Hyperbolicity, Manifolds, Tangency of manifolds, Transversality, Spectrum of Lyapunov exponents

\section{INTRODUCTION}

Typical chaotic attractors of dynamical systems can be divided into robust hyperbolic attractors, almost hyperbolic (quasihyperbolic) attractors and socalled quasiattractors(Shilnikov, 1993; Afraimovich and Shilnikov, 1983; Plykin, 1980). Such a classification of attractors is a natural consequence of the rigorous mathematical analysis of the structure and properties of dynamical chaos. However, from the experimental point of view, it has not been accepted as significant. Studies during recent years have convincingly shown that properties of hyperbolic, quasihyperbolic attractors and quasiattractors are essentially different. It manifests itself in the differences between their characteristics that are obtained in numerical experiments (Anishchenko and Strelkova, 1998).

The aim of the present paper is to study quasihyperbolic attractors and quasiattractors in two-dimensional invertible maps in order to obtain some characteristic properties which will allow one to diagnose exactly their difference.

The paper is organized as follows. In Sections 2 and 3 of the paper we give definitions and formulate the main properties of robust hyperbolic, quasihyperbolic attractors and quasiattractors. Section 4 illustrates transversality of intersection of manifolds of saddle points as a principal property of hyperbolic and quasihyperbolic attractors. A violation of the transversality leads to the birth of a quasiattractor. In Sections 5 and 6 we study the

* Corresponding author. 
regularities in the behavior of manifolds of chaotic trajectories in the Henon attractor, Lozi attractor and in the map on the torus. We calculate the largest Lyapunov exponent as a function of system parameter variation. The criteria, which allow one to diagnose the type of an attractor in experiments, are formulated. And finally (Section 7), we discuss the results of investigations and present the main conclusions on this work.

\section{ROBUST HYPERBOLIC AND QUASIHYPERBOLIC ATTRACTORS}

The property of hyperbolicity of a chaotic attractor means that all trajectories are of the same saddle type, i.e., each trajectory has transversal stable and unstable manifolds and their dimensionality is the same for all trajectories. For hyperbolic attractors both saddle periodic and robust homoclinic orbits are everywhere dense. They satisfy the axiom A of Smale and are structurally stable (robust), i.e., they are preserved under perturbations. Since all the trajectories of the hyperbolic attractor belong to the same saddle type, its structure is homogeneous. A neighborhood of any point of the attractor has the same geometry. There are examples of conservative systems for which all trajectories of the phase space are hyperbolic. These are the so-called C-systems of Anosov (Anosov, 1967; Arnold, 1978). But for conservative systems we cannot speak of an attractor. As for dissipative systems, for the present time no mathematical model in the form of differential equations or maps is known for which the existence of the robust hyperbolic attractor was strictly proved. However, there are a number of examples of almost hyperbolic attractors. Their difference from robust hyperbolic attractors is a local violation of homogeneity due to the presence of singular phase trajectories belonging to another saddle type. This can be saddle equilibrium states, which have a different dimension of manifolds, or separatrix circuits. As an example we can mention here almost hyperbolic Lorenz attractor, which includes a denumerable set of separatrix loops of the saddle equilibrium state (Williams, 1977; Afraimovich et al., 1977; Shilnikov, 1980). The homogeneity of the attractor can also be violated as a result of the birth of nonrobust homoclinic trajectories. In order for the attractor to be almost hyperbolic such trajectories must have not only a zero measure on the attractor but should also be "non-dangerous", i.e., their appearance and disappearance should not lead to the birth of stable trajectories and affect the structure of the chaotic hyperbolic set. Attractors of the similar type can be easily realized in two-dimensional (2-D) maps composed by a special way (Belykh, 1995). Thus, the Lozi attractor (Lozi, 1978) and the Belykh's attractor (Belykh, 1982) are well-known examples of quasihyperbolic attractors.

\section{QUASIATTRACTORS}

Most chaotic attractors observed in various dynamical systems are neither robust hyperbolic nor almost hyperbolic attractors. They are the so-called quasiattractors. Such attractors enclose non-robust singular trajectories that are "dangerous". This can be separatrix loops of saddle-focuses or homoclinic curves of saddle cycles in the moment of tangency of their stable and unstable manifolds. In the neighborhood of such trajectories there appears a map of "Smale's horseshoe"-type that contains both non-trivial hyperbolic subset of trajectories and a denumerable subset of stable periodic orbits (see Shilnikov's theorem (Shilnikov, 1963; Gavrilov and Shilnikov, 1972; 1973) and Newhouse's theorem (Newhouse, 1980)). The strange attractor proves to be "holed" by a set of basins of attraction of different periodic orbits. The basins of attraction of stable cycles are very narrow and so mixed in the phase space that it is not always possible to detect them in numerical experiments. In practice, even very small noise "fills up the holes" in the quasiattractor. By virtue of these reasons, the whole attracting set of trajectories including a subset of both chaotic and stable periodic trajectories is considered as the unified limit attracting set called the quasiattractor. 


\section{QUALITATIVE DIFFERENCES IN THE BEHAVIOR OF MANIFOLDS OF SADDLE EQUILIBRIUM STATES IN THE HENON AND LOZI MAPS}

2-D invertible maps are diffeomorphisms and can be considered as the models of Poincare maps in the section of 3-D flows. Hence, the well-known Henon map

$$
\begin{aligned}
& x_{n+1}=a-x_{n}^{2}+y_{n}, \\
& y_{n+1}=b x_{n},
\end{aligned}
$$

simulates a 3-D flow system with a chaotic attractor of the Rössler-type or similar to the attractor in Anishchenko-Astakhov's oscillator (Anishchenko, 1995). The Lozi map that is similar in its form to the Henon map but is characterized by a piecewiselinear non-linearity (Lozi, 1978)

$$
\begin{aligned}
& x_{n+1}=1-a\left|x_{n}\right|+y_{n}, \\
& y_{n+1}=b x_{n},
\end{aligned}
$$

has the attractor which, in its properties, is close to the Lorenz attractor. It has been proved theoretically that the Henon attractor is an example of a quasiattractor, while the Lozi map is an example of a quasihyperbolic attractor. These rigorous results will be the basis of the studies presented in this paper.

The behavior of manifolds of saddles plays a principal role in forming chaotic attractors. In 2-D maps manifolds of saddles are 1-D curves and their behavior can be analyzed numerically. Chaotic attractors are located along the unstable manifolds of saddle cycles repeating their form. In a dissipative map of the plane the unstable manifolds of saddles, as well as the chaotic attractor itself, must "be packed" in some bounded region of the phase plane. In consequence of this, when giving the righthand parts of the map by smooth functions, the unstable manifolds inevitably undergo a bending in the form of "horseshoe". This fact leads to "dangerous" tangencies between the stable and unstable manifolds and, respectively, to the quasiattractor.

Let us illustrate the mentioned above using the Henon map. Figure 1(a) shows the behavior of the manifolds of a saddle fixed point (period-1 cycle) for map (1). Manifolds of saddle cycles of other periods behave qualitatively the same way. It has been proved that homoclinic tangencies are everywhere dense in the parameter space (Newhouse, 1980) and the quasiattractor is a typical limit set for the Henon map and similar to it maps. However, "dangerous" tangencies can be avoided if one uses piecewise-smooth functions for a map (Banerjee et al., 1998). According to this principle, the Lozi map (2) was constructed.

The behavior of the manifolds of a saddle fixed point for map (2) is shown in Fig. 1(b). It is seen that

(a)

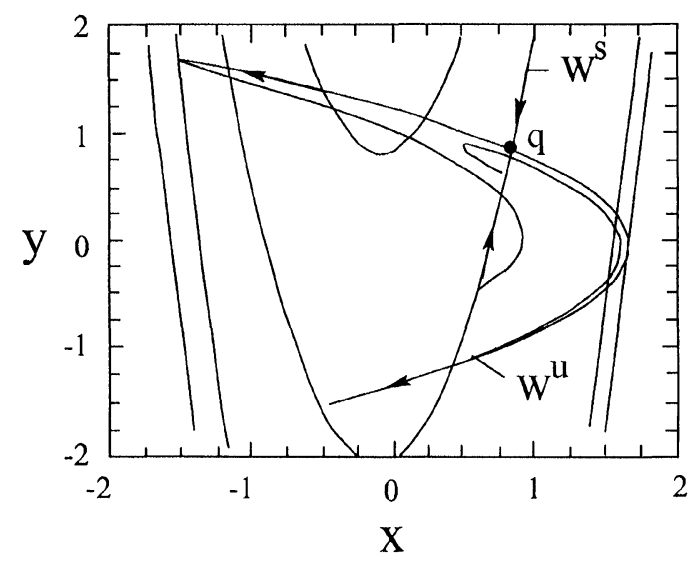

(b)

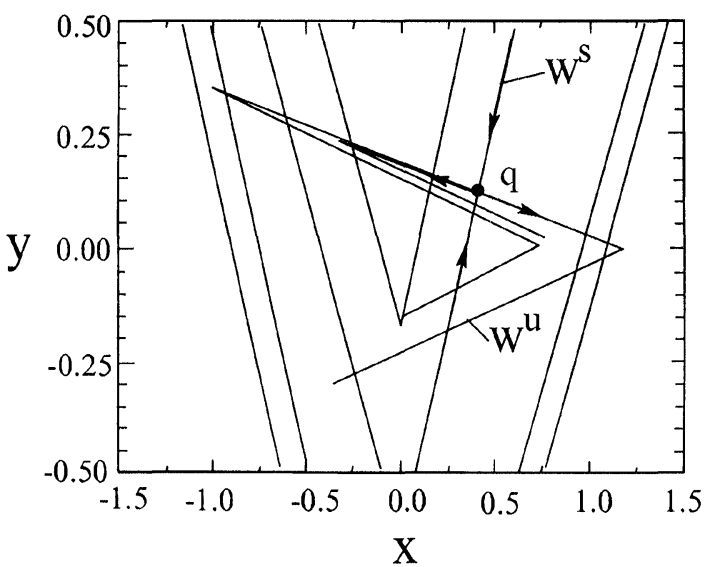

FIGURE 1 Stable and unstable manifolds of saddle points in the Henon map and the Lozi map. (a) The manifolds of the saddle in map (1) for $a=1.3$ and $b=0.3$ and (b) the manifolds of the saddle in map (2) for $a=1.7$ and $b=0.3$. 
at the intersection of the stable and unstable manifolds the angle between them is not equal to zero. The intersection of the manifolds is everywhere transversal and the appearance of homoclinic trajectories does not lead to the birth of stable periodic orbits. The hyperbolic chaotic set remains the only attracting limit set and in this case we speak of the quasihyperbolic attractor.

\section{NUMERICAL STUDIES OF THE PROPERTIES OF QUASIHYPERBOLIC ATTRACTORS AND QUASIATTRACTORS}

The most direct method for numerical analysis, whether a trajectory on the chaotic attractor is hyperbolic or not, is the exploration of the behavior of the angle between stable and unstable manifolds of the chaotic trajectory when moving on the attractor. The algorithm of such an investigation was proposed in (Lai et al., 1993) where it was used in general to analyze hyperbolicity of chaotic saddles. This procedure consists in the forward and backward transformation of an arbitrary vector by a linearized evolution operator along the trajectory considered. It allows one to find the angle between the directions of stability and instability for various points of the trajectory on the attractor. Naturally, such calculations can be more easily carried out for 2-D invertible maps.

We shall calculate angles between manifolds of a chaotic trajectory for different points of the attractor and analyze their statistics. Based on these results one can conclude whether the attractor is hyperbolic or one deals with the quasiattractor. We shall also calculate dependencies of Lyapunov exponents of chaotic motions on the controlling parameters.

A typical example for map (1) distribution of the probabilities of the angle between the manifolds of a chaotic trajectory $P(\phi)$ on the quasiattractor is shown in Fig. 2(a). The probability of the angle in the neighborhood of zero is finite. This fact indicates the presence of tangency points of manifolds. Practically it means the existence of non-robust (a)

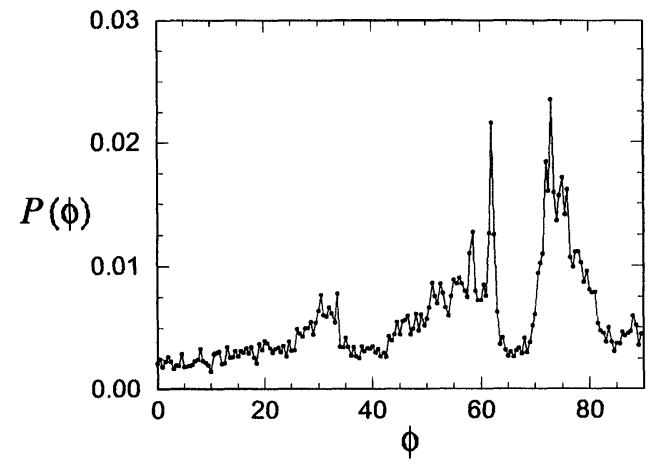

(b)

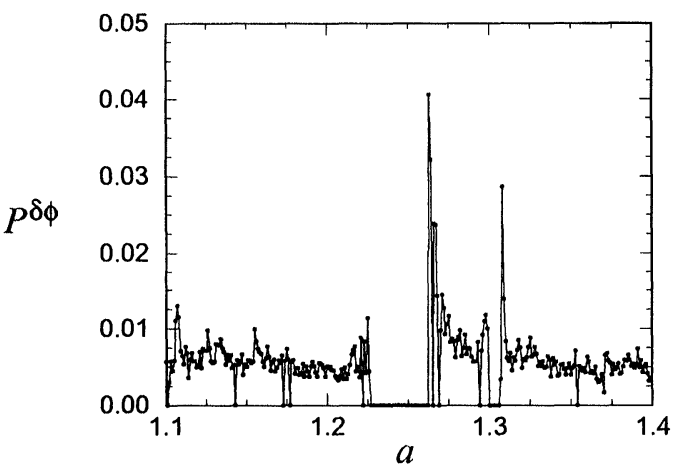

(c)

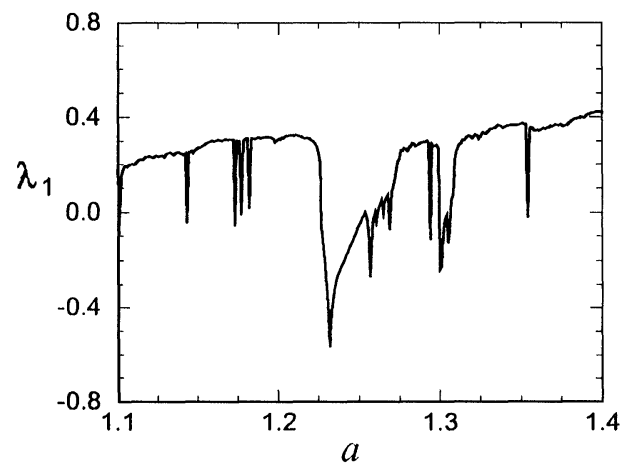

FIGURE 2 Calculation results of the characteristics for the Henon attractor (a) A distribution of the probabilities of the angle $\phi$ between stable and unstable manifolds for $a=1.179$ and $b=0.3$, (b) the probability that the angle $\phi$ falls within the interval $0<\phi \leq 1^{\circ}\left(\delta \phi=1^{\circ}\right)$ versus parameter $a$ for $b=0.3$ and (c) a dependence of the largest Lyapunov exponent on parameter $a$ for $b=0.3$.

homoclinic curves of saddle cycles along which manifolds of the cycles approach each other tangentially. The points of the chaotic trajectory falling within small enough neighborhoods of such curves contribute to the probability that the angle $\phi$ falls within the neighborhood of zero. 
In order to imagine visually how much quasiattractors are typical for map (1) and, respectively, hyperbolic attractors are not characteristic, we calculated the probability $P^{\delta \phi}$ that the angle $\phi$ falls within a small neighborhood of zero $\left(\delta \phi=1^{\circ}\right)$ as a function of the controlling parameter $a$. The calculation algorithm was constructed in such a way that if at a certain parameter value the trajectory does not have the unstable manifold, the probability $P^{\delta \phi}$ is equal to zero. This dependence is presented in Fig. 2(b). Except of a set of the parameter values corresponding to non-zero probabilities of falling within the neighborhood of zero, there is a denumerable set of the parameter values for which the probability $P^{\delta \phi}(a)$ is equal to zero. One may assume that for certain of these parameter values the attractor of system (1) is hyperbolic (it is known that such points exist). However, almost for all of the examined cases the fact that $P^{\delta \phi}(a)$ is equal to zero corresponds to windows of stability of periodic trajectories.

Figure 2(c) displays a dependence of the largest Lyapunov exponent of system (1) on the variation of parameter $a$ and at fixed initial conditions. Such a rugged dependence with a set of jumps to the regions of negative values, which correspond to periodic windows, is typical for systems with quasiattractors. The behavior of such systems including averaged characteristics of motions is very sensitive to small parameter variations and perturbations of the right-hand parts of equations.

For the Lozi map (2) whose attractor is quasihyperbolic the examined characteristics are qualitatively different from those for the Henon map.

Figure 3(a) shows a typical example for map (2) distribution of the angle $\phi$ between the directions of stable and unstable manifolds of a chaotic trajectory. As seen from the picture, there is some minimal value of the angle and it is bounded far away from zero. This minimal value $\phi_{\min }$ depends obviously on the parameters of the map. Such a dependence on parameter $a$ for $b=0.3$ is given in Fig. 3(b). One can see that for complete range of the parameter $a$ values where the chaotic attractor exists the minimal angle between the directions of the stable and unstable manifolds of the phase trajectory is $>40^{\circ}$ and is never equal to 0 . Manifolds of chaotic trajectories in the Lozi attractor behave the same way as manifolds of saddle cycles, i.e., they are always transversal. Figure 3(c) shows a dependence of the largest Lyapunov exponent of map (2) on parameter $a$.

(a)

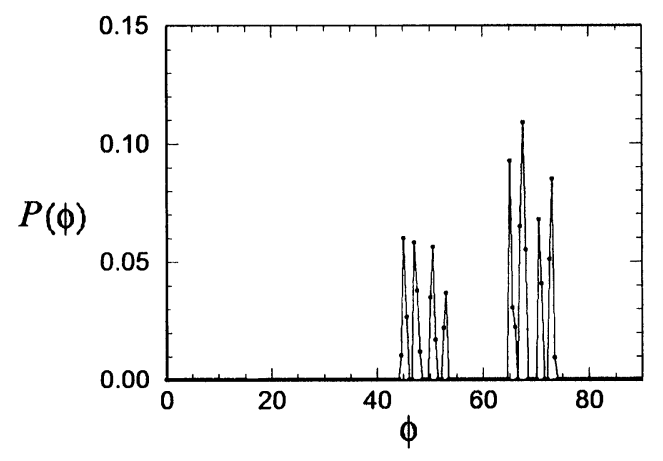

(b)

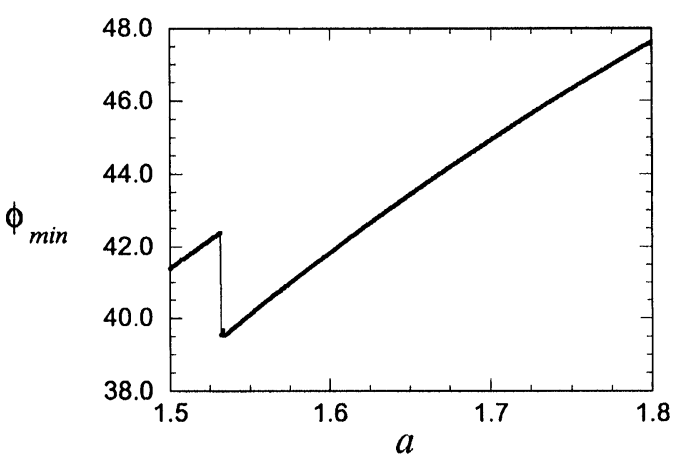

(c)

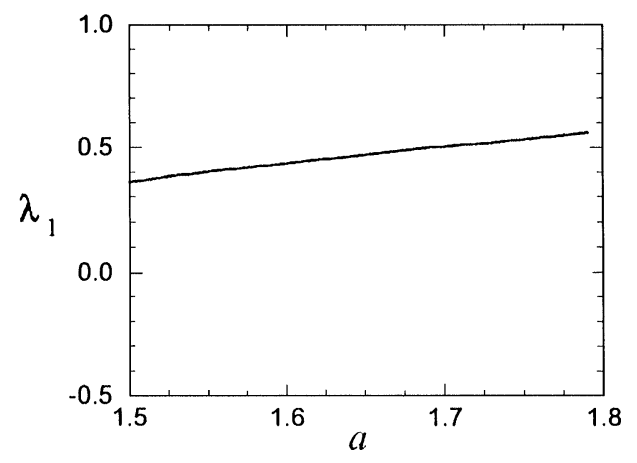

FIGURE 3 Calculation results of the characteristics for the Lozi attractor. (a) A distribution of the probabilities of the angle $\phi$ between stable and unstable manifolds for $a=1.7$ and $b=0.3$, (b) a plot of the minimal angle $\phi_{\min }$ versus parameter $a$ for $b=0.3$ and (c) a dependence of the largest Lyapunov exponent on $a$ for $b=0.3$. 
Within the limits of calculation errors this dependence is a smooth positive definite function.

The differences between the characteristics for the Henon attractor and the Lozi attractor, which are shown in Figs. 1-3, are evident. Therefore, these characteristics can be used to diagnose the types of attractors in those systems whose theoretical study is difficult.

\section{QUASIHYPERBOLIC ATTRACTOR IN THE DISSIPATIVE MAP ON A TORUS}

One more way to avoid "dangerous" tangencies of manifolds in a 2-D map is to consider a map on a torus. In this case manifolds of a saddle will bend around the torus surface intersecting transversally each time. In such a situation any non-robust homoclinic orbits are excluded although the map can be given by smooth functions. Here the absence of homoclinic tangencies means robust hyperbolicity. So, "cat map" described by Anosov (1967) is a well-known example of the robust hyperbolic system. It has the following form:

$$
\begin{aligned}
& x_{n+1}=x_{n}+y_{n}, \bmod 1, \\
& y_{n+1}=x_{n}+2 y_{n}, \bmod 1 .
\end{aligned}
$$

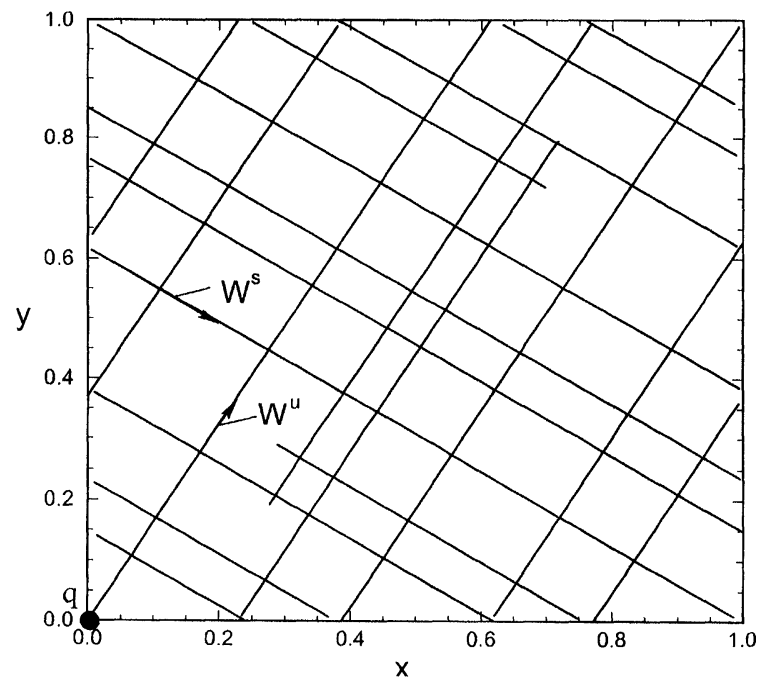

FIGURE 4 The stable and unstable manifolds of the saddle point in "cat map" (3).
The behavior of the manifolds of a saddle for map (3) is shown in Fig. 4. However, map (3) is conservative and we cannot speak of an attractor. In order to deal with an attractor one can introduce a small perturbation to map (3). It will make the map on the torus dissipative but keep it invertible. For instance,

$$
\begin{aligned}
& x_{n+1}=x_{n}+y_{n}+\delta \sin 2 \pi y_{n}, \bmod 1, \\
& y_{n+1}=x_{n}+2 y_{n}, \bmod 1
\end{aligned}
$$

Such a modified "cat map" was considered in (Farmer et al., 1983; Sinai, 1972). For $\delta<\frac{1}{2} \pi$, map (4) is dissipative and a diffeomorphism of the torus. The manifolds of a saddle slightly distort their shapes compared to (3) but their behavior remains qualitatively the same. It is probable that the attractor observed in (4) in the region of invertibility of the map is robust hyperbolic but we do not know a rigorous proof of this fact (Belykh, 1995).

Let us calculate all the characteristics that are represented in Figs. 2 and 3.

Figure 5 illustrates the corresponding results for map (4) on the torus. They repeat qualitatively the results obtained for the Lozi map (2). A typical example for map (4) distribution of the angle $\phi$ between the directions of manifolds for $\delta<\frac{1}{2} \pi$ (in the region of invertibility of the map) is presented in Fig. 5(a). Figure 5(b) shows a dependence of the minimal angle $\phi_{\min }$ on map parameter $\delta$. Finally, the dependence of the largest Lyapunov exponent of a chaotic trajectory on the parameter is plotted in Fig. 5(c). As for the Lozi map, the minimal angle is nowhere equal to zero and the dependence of the Lyapunov exponent versus the parameter is smooth. If one considers dependences of some other averaged characteristics of chaotic attractors on the parameter, then for (2) and (4) they will be smooth. Therefore, the behavior of the system possessing hyperbolic or almost hyperbolic attractors in some region of the parameter space is stable with respect to small changes in parameters. Nonrobustness of almost hyperbolic attractors that is connected with appearance and disappearance of 
(a)

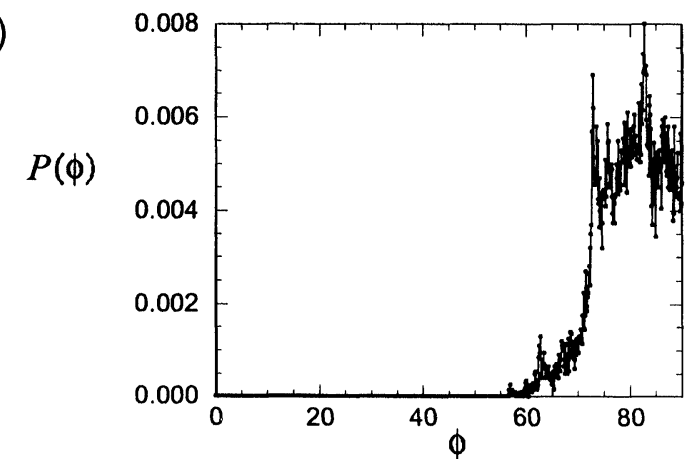

(b)

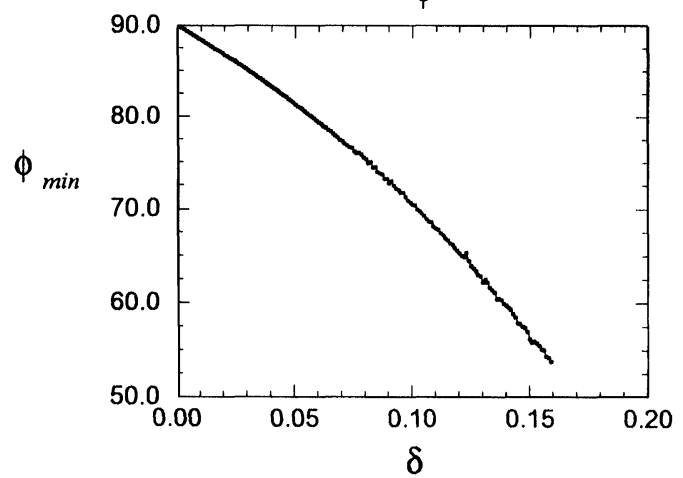

(c)

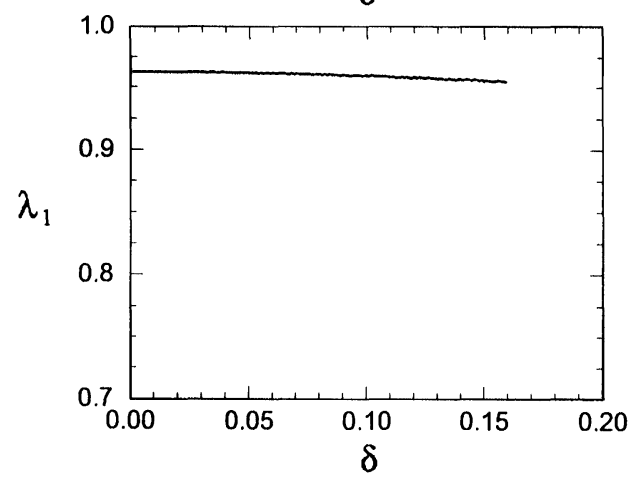

FIGURE 5 Calculation results of the characteristics for the attractor in map (4). (a) A distribution of the probabilities of the angle $\phi$ between stable and unstable manifolds for $\delta=0.15$, (b) a plot of the minimal angle $\phi_{\min }$ versus parameter $\delta$ and (c) a dependence of the largest Lyapunov exponent on $\delta$.

singular trajectories does not play an important role here. As it has already been mentioned, these singular trajectories being "non-dangerous" and having a zero measure in phase space do not affect the averaged characteristics of the motion. Thus, from the experimental point of view, one can classify the chaotic attractor in map (4) as quasihyperbolic.

\section{CONCLUSIONS}

As a result of the investigations one can formulate a number of properties of quasihyperbolic attractors which are available for numerical calculations and can serve as diagnostic criteria to determine the type of a chaotic attractor. For quasihyperbolic attractors the following properties are characteristic. The probability density of the angle between stable and unstable separatrixes of a chaotic trajectory of the attractor is strictly equal to 0 in the neighborhood of a zero value of the angle, i.e., $p(\phi)=0$ for $\phi \simeq 0$ and $p(\phi)>0$ for $0<\phi \leq \phi_{0}$ (see Fig. 3(a)). When varying the system controlling parameter, the minimal angle $\phi_{\min }$ between the manifolds does not approach zero, $\phi_{\min }>0$. The dependence of the largest Lyapunov exponent $\lambda_{1}$ on the parameter in the region where the attractor exists is a smooth positive definite function, $\lambda_{1}(a)>0, a_{1} \leq a \leq a_{2}$.

It is absolutely clear that transversality of manifolds is a major condition and all other characteristics are its natural consequence. Therefore, for two-dimensional maps one can consider the transversality as a necessary condition for the attractor to be quasihyperbolic. However, as our investigations have shown, in the case of differential dynamical systems in $\mathfrak{R}^{3}$ when it is impossible to calculate the angle between manifolds, one can differentiate quasihyperbolic attractors from quasiattractors using the characteristics mentioned above. We also used (in addition to the characteristics described in the paper) such a property of quasihyperbolic attractors as a typical form of autocorrelation function (ACF). For the case of the quasihyperbolic attractor ACF decreases according to the law $\exp \left(-\lambda_{1} \tau\right)$ where $\lambda_{1}$ is the positive Lyapunov exponent. As a consequence, the power spectrum of quasihyperbolic attractors does not contain any pronounced peaks and depends smoothly enough on the frequency.

This work was supported in part by the Russian Committee of Higher Education (grant No. 97-08.3-47) and the Russian Foundation of Basic Research (grant No. 98-02-16531). 


\section{References}

Afraimovich, V., Bykov, V. and Shilinikov, L. (1977). On the appearance and structure of Lorenz attractor. DAN USSR, 234, 336-339 (in Russian).

Afraimovich, V. and Shilnikov, L. (1983). Strange attractors and quasiattractors. In: Nonlinear Dynamics and Turbulence (G.I. Barenblatt, G. Iooss and D.D. Joseph, Eds.). Pitman, Boston, London, Melbourne, pp. 1-51.

Anishchenko, V. (1995). Dynamical Chaos - Models and Experiments. World Scientific, Singapore, p. 400.

Anishchenko, V. and Strelkova, G. (1998). Irregular attractors. Discrete Dynamics in Nature and Society, 2(1), 53-72.

Anosov, D. (1967). Geodesic flows on closed Riemann manifolds of negative curvature. In: Proc. V.S. Steklov Math. Inst., 90, 3-209.

Arnold, V. (1978). Additioinal chapters to the Theory of Ordiniary Differential Equations. Nauka, Moscow (in Russian).

Banerjee, S., Yorke, J. and Grebogi, C. (1998). Robust Chaos. Phys. Rev. Lett., 80(14), 3049-3052.

Belykh, V. (1982). Models of discrete systems of phase locking. In: Phase Locking Systems (L.N. Belyustina and V.V. Shakhgil'dyan, Eds.). Radio i Svyaz, Moscow, pp. 161-176 (in Russian)

Belykh, V. (1995). Chaotic and strange attractors of a twodimensional map. Math. Sbornik, 186(3), 311-326. (in Russian).
Farmer, J., Ott, E. and Yorke, J. (1983). The dimension of chaotic attractors. Physica $D, 7,153$.

Gavrilov, N. and Shilnikov, L. $(1972 ; 1973)$. About threedimeinsional dynamical systems close to nonrobust homoclinic curve. Math. Sbornik, 88(130), No. 8, 475-492; Math. Sbornik, 90(132), No. 1, 139-156.

Lai, Y., Grebogi, C. and Yorke, J. (1993). How often are chaotic saddles nonhyperbolic? Nonlinearity, 6, 779-797.

Lozi, R. (1978). Un Attracteur Etrange du Type Attracteur de Henon. Journal de Physique, 39(C5), 9-10.

Newhouse, S. (1980). Ann. of N.Y. Acad. Sci., 357, 292-299.

Plykin, R. (1980). About hyperbolic attractors of diffeomorphisms. Uspekhi Math. Nauk, 35(3), 94-104 (in Russian).

Shilnikov, L. (1963). On some cases of birth of periodic motions from singular trajectories. Math. Sb., 61(104), 443-466 (in Russian).

Shilnikov, L. (1980). The theory of bifurcations and the Lorenz model. In: The Hopf Bifurcation and Its Applications (J. Marsden and M. McCracken, Eds.). Mir, Moscow, pp. 317 335 (in Russian).

Shilnikov, L. (1993). Strange attractors and dynamical models. Journal of Circuits, Systems, and Computers, 3(1), 1-10.

Sinai, Y. (1972). Gibbs measure in ergodic theory. Russ. Math. Surveys, 4, 21-64 (in Russian).

Williams, R. (1977). The structure of Lorenz attractors. Lect. Notes in Math., 615, 94-112. 


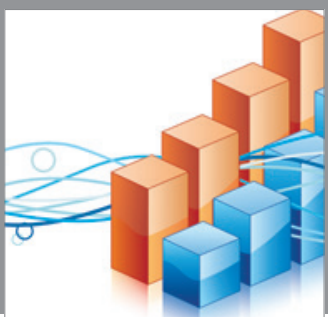

Advances in

Operations Research

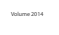

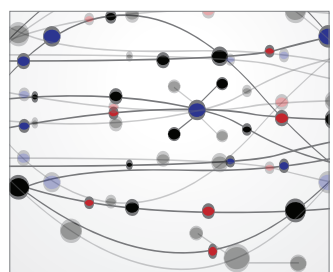

\section{The Scientific} World Journal
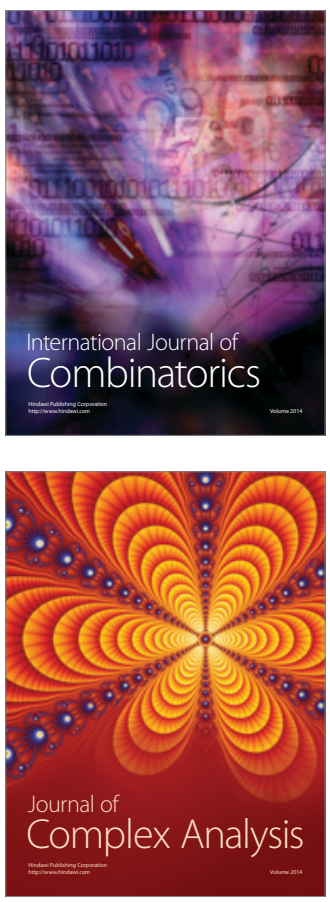

International Journal of

Mathematics and

Mathematical

Sciences
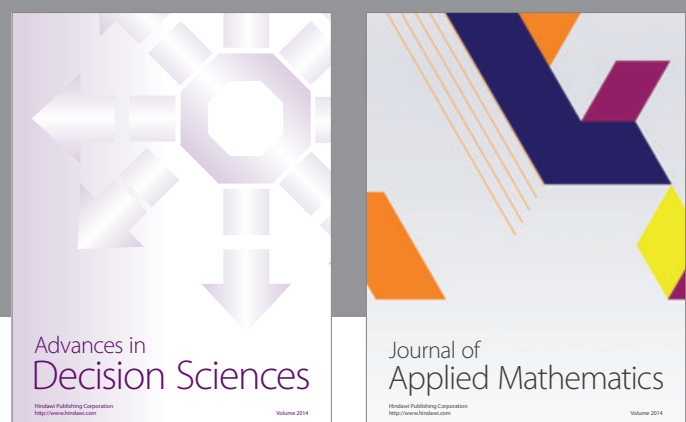

Journal of

Applied Mathematics
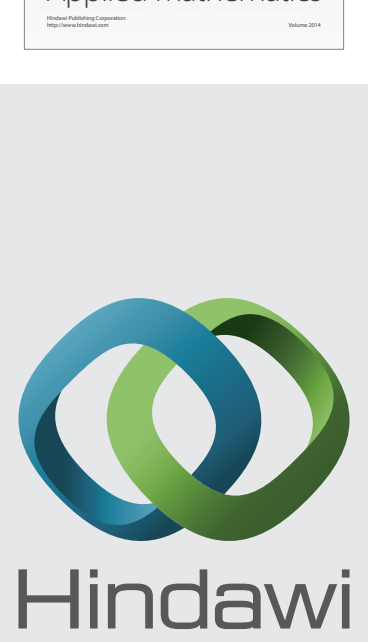

Submit your manuscripts at http://www.hindawi.com
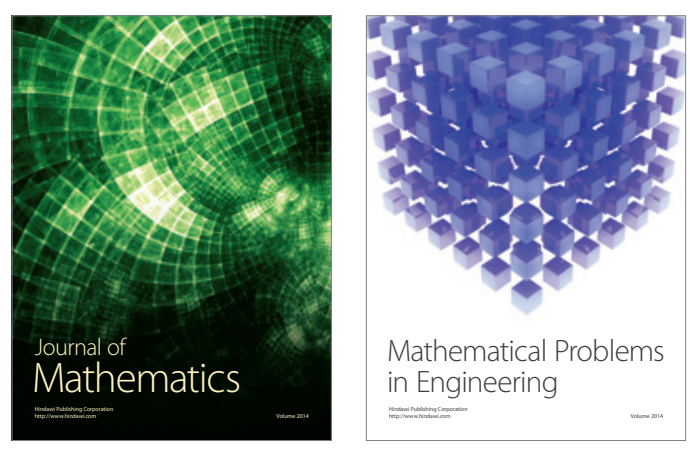

Mathematical Problems in Engineering
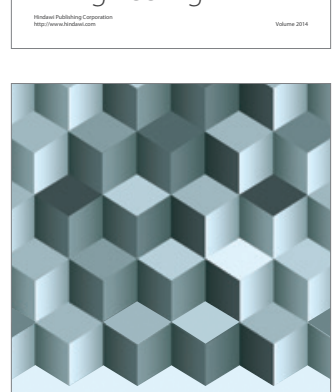

Journal of

Function Spaces
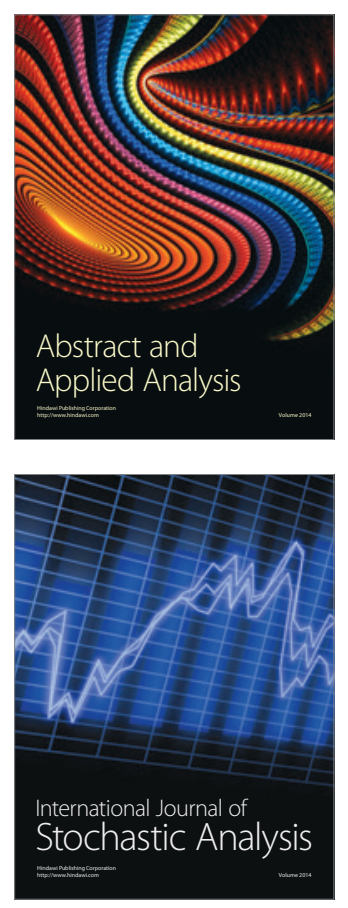

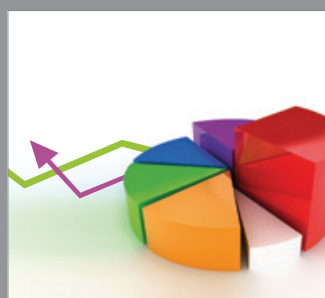

ournal of

Probability and Statistics

Promensencen
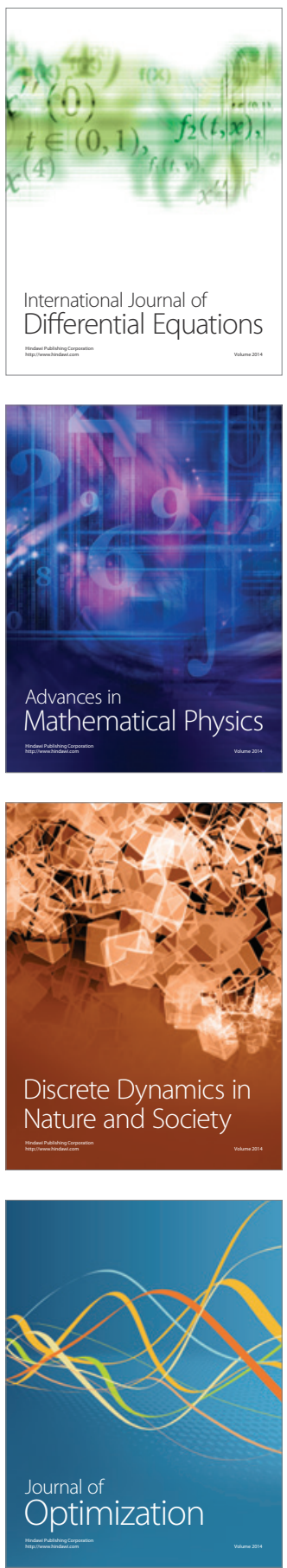